\title{
Linx
}

Revue des linguistes de l'université Paris X Nanterre

$11 \mid 1999$

Typologie des langues, universaux linguistiques

\section{Thématisation et préconstruit, la structure des relatives en nêlêmwa : éléments de typologie}

Isabelle Bril

\section{(2) OpenEdition}

Journals

Édition électronique

URL : http://journals.openedition.org/linx/889

DOI : $10.4000 /$ linx. 889

ISSN : 2118-9692

Éditeur

Presses universitaires de Paris Nanterre

\section{Édition imprimée}

Date de publication : 1 décembre 1999

Pagination : 105-124

ISSN : 0246-8743

\section{Référence électronique}

Isabelle Bril, «Thématisation et préconstruit, la structure des relatives en nêlêmwa : éléments de typologie », Linx [En ligne], 11 | 1999, mis en ligne le 29 juin 2012, consulté le 01 mai 2019. URL : http:// journals.openedition.org/linx/889; DOI : 10.4000/linx.889

Ce document a été généré automatiquement le 1 mai 2019.

Département de Sciences du langage, Université Paris Ouest 


\title{
Thématisation et préconstruit, la structure des relatives en nêlêmwa ${ }^{1}$ : éléments de typologie
}

\author{
Isabelle Bril
}

1 Je retiendrai comme définition d'une relative la détermination d'un groupe nominal par une proposition, abstraction faite de la présence ou de l'absence de relateurs. Cette définition est donc fonctionnelle.

2 Les langues de Nouvelle-Calédonie se signalent tout d'abord par l'absence de pronoms relatifs, mais des morphèmes apparaissent toutefois à la jonction entre le nom et sa détermination propositionnelle : il s'agit généralement soit d'un déterminant déictique ou anaphorique, soit d'un morphème dérivé d'articles définis ou indéfinis (cemuhî, Rivierre:1980).

3 Du point de vue typologique, l'absence de relatif est souvent attestée ${ }^{2}$, de même que la présence d'un déterminant anaphorique faisant fonction de relateur, c'est même l'une des origines possibles des « pronoms relatifs » dans des langues non apparentées.

4 Qu'en est-il en nêlêmwa ? L'ordre général de la détermination nominale, relatives incluses, est de type déterminé - déterminant. Il n'y a pas de hiérarchie des fonctions syntaxiques relativisables dans cette langue : tout terme peut être déterminé par une relative, quelle que soit sa fonction syntaxique (actant ou circonstant) et sa marque casuelle (absolutif, ergatif). La distinction entre relative déterminative et relative restrictive n'est pas opératoire en nêlêmwa, ni dans les langues kanak en général. Toutefois, on distingue deux types de "propositions relatives » en nêlêmwa, selon le type de relateur : celles qui sont construites avec le morphème de thématisation xe (1) et celles dont le relateur est un déterminant déictique ou anaphorique (2) et (3). 
$\begin{array}{llllll}\text { (1) na fhe } & \text { pwaxi-n } & x e & i & \text { khîlu. } \\ \text { 1SG amener } & \text { enfant-POSS.3SG } & \text { CONN } & \text { 3SG malade }\end{array}$

'J'amène son enfant qui est malade.' (ou) 'J'amène son enfant, il est malade.'

L'énoncé (1) peut aussi être traduit par une thématisation.

5 - déterminant déictique

\begin{tabular}{|l|l|l|l|l|l|l|}
\hline$(2)$ & $n a$ & fhe & [pwaxi-n & hleny & khîlû $]$ & dame. \\
\hline & $1 \mathrm{sg}$ & amener & enfant-Poss.3SG & ce.DEICT & être malade & en haut ici \\
\hline \hline
\end{tabular}

6 - déterminant anaphorique

(3)
na fhe
[pwaxi-n
bai
khîlî ]
dame.
1SG amener enfant-POSS.3SG
ce.ANAPH
être malade
en haut ici
'J'ai amené son enfant qui est malade.'

7 Les relatives construites avec xe constituent la première mention de la détermination, elles identifient ou qualifient le terme déterminé; ce morphème est associé au mode affirmatif, non virtuel; si le mode de l'énoncé est virtuel / hypothétique, c'est le marqueur oqui apparaît (cf $\S 4$.).

8 Les relatives associées à un déterminant déictique ou anaphorique réfèrent à une détermination préconstruite, déjà connue par le contexte ou identifiable par la situation ; ce déterminant est post-nominal et s'accorde en nombre avec l'antécédent.

9 La comparaison des énoncés (1) d'une part et (2 \& 3) d'autre part, montre que le type de relation est différent: en (1), xe marque l'articulation d'un thème et d'un rhème, la proposition relative identifie l'enfant et le pronom sujet $i[x e \boldsymbol{i}$ khîlû] est obligatoire; en revanche, le pronom sujet est effacé en (2) et (3) où l'enfant malade est déjà référentiel. D'autre part, la présence du directionnel dame « en haut vers ici » en clôture d'énoncé en (2 \& 3) signale que la relation entre le nom et sa détermination forme un bloc déterminatif. Cette construction serait impossible en (1) du fait de la démarcation opérée par $x e$ entre les deux segments, le directionnel dame ne peut porter sur un verbe statif [* na fhe pwaxi-n xe $i$ khîlû dame ], il ne pourrait porter que sur le verbe fhe " amener " [na fhe damepwaxi-n $x e$ i khîlû ].

10 La construction des relatives est donc liée aux étapes de la construction de la détermination dans le discours. Enfin, la position du relateur ne peut être vide, cette place doit être saturée par un des deux types de morphèmes mentionnés pour structurer la relation entre l'antécédent et la relative.

\section{1. xe : morpheme plurifonctionnel}

11 Outre la thématisation (4b)et sa fonction de démarcation dans certains types de relatives, le morphème $x e$ a d'autres fonctions et marque certaines complémentations verbales $(7$, 8). Quel est le dénominateur commun à toutes ces fonctions? 
12 Qu'il s'agisse de détermination verbale (complémentation) ou nominale (relativisation), xe est associé à la construction de la détermination, et cette détermination identifie le terme.

\subsection{Xe : marqueur de thématisation discursive}

L'ordre de l'énoncé neutre est (prédicat / argument) (4a). La thématisation (4b) inverse cet ordre [argument $x e$ prédicat], l'argument devient le thème marqué par $x e$.

14 - Ordre neutre :
(4a) $i$
3SG
axi-e
$a$
thaamwa
ena.
AGT femme

\section{ce.DEICT}

'Cette femme le voit.'

15 - Argument thématisé :
(4b) thaamwa
ena

femme ce.DEICT CONN 3SG voir.TR-3SG

'Cette femme, elle le voit.'

(La thématisation de l'agent induit l'effacement de la marque d'agent.)

16 L'organisation thème / rhème s'applique aussi aux relations interpropositionnelles, telles que l'organisation entre apodose et protase dans les subordonnées hypothétiques ${ }^{4}$. Si la protase est choisie comme le terme de départ thématique de la relation, xe apparaît ; on oppose ainsi (5a) et (5b) :

17 - apodose -protase
a
yo tâlâ-e
$o$
i
thoogi-yo.
INJ.NÉG 2SG écouter-3SG si 3SG
appeler-2SG
'N'écoute pas s'il t'appelle.'

18 - protase $-x e$ - apodose
(5b)
thoogi-yo xe $a$
yo tâlâ-e.
si 3SG appeler-2SG CONN INJ.NÉG 2SG écouter-3SG
'S'il t'appelle, n'écoute pas.'

Le thème est encore une proposition entière en (6),xemarque alors une articulation logique interpropositionnelle de type séquentiel :

$\begin{array}{llllll}i & \text { khabwe } & x e & \text { io } & i & \text { diya. } \\ \text { 3SG } & \text { dire } & \text { CONN } & \text { FUT } & \text { 3SG } & \text { faire }\end{array}$

'Il l'a dit, (alors) il le fera.' (ou) 'Il a dit qu'il le fera.'

On voit se profiler le glissement de la thématisation vers la complémentation: le morphème démarcatif xe qui articule la relation thème / rhème (« il l'a dit, il le fera ») glisse vers l'hypotaxe (« il a dit qu'il le fera »). 


\subsection{Xe : marqueur de complémentation ou connecteur}

21 Xe construit la complémentation de certains verbes tels que axe 'voir' or kaxaak 'être sûr' et parfois celle du verbe khabwe 'dire' dans le discours indirect. Il est associé à des énoncés déclaratifs et factifs.

$$
\begin{array}{llllll}
i & a x e & x e & x u & j e u k & t a n . \\
\text { 3SG } & \text { voir } & \text { CONN } & \text { PERF } & \text { être proche } & \text { nuit }
\end{array}
$$

'Il voit que la nuit approche.'

$\begin{array}{llllll}\text { (8) na kaxaak } & \text { xe } & \text { na } & \hat{a} & \text { Pum. } \\ \text { 1SG être sûr CONN } & \text { 1SG } & \text { aller } & \text { Poum } \\ \\ \text { Je suis sûr d'aller à Poum.' }\end{array}$

Le morphème xe est également associé à des locutions de subordination telles que uya da $x e$ 'jusqu'à ce que' (9) et puxet xe'parce que' (10) où il garde sa fonction de thématisation et de démarcation entre le thème et le prédicat:

(9)

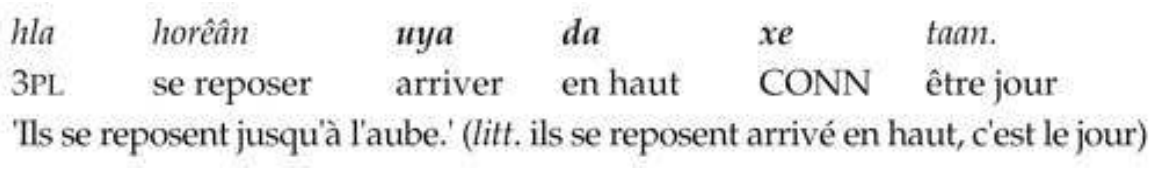

En (10), xedémarque deux thèmes successifs : la proposition $i$ cêe biin constitue le premier thème, le second thème est le nom puxe-t 'raison, origine, base', qui introduit la subordonnée explicative.
(10) $[i \quad$ cêe biin
xe [puxet xe $[i$ côe khillu $]]]$.
3SG très être faible CONN raison CONN 3SG très être malade
'Il est très faible parce qu'il est très malade.'
(litt. il est très faible, la raison c'est qu'il est très malade).

\section{2. xe: « relatif »}

Dans les propositions relatives, $x e$ a plutôt la fonction d'un connecteur signalant que la détermination nominale qui suit constitue une identification. Toutes les fonctions syntaxiques peuvent être relativisées avec $x e$.
(11) fo agu xe hla
uya.
il y a gens
CONN 3PL
arriver
'Il y a des gens qui arrivent.' (ou) 'Il y a des gens, ils arrivent.'

En (12), la première occurrence de xe marque la thématisation de l'adverbe kôôbwan, la deuxième occurrence articule la relative.
kôobruan xe
$i$ fawulu hliibai
thaamwa $x e$
hli haga.
Hier CONN 3SG parlerà ces.2.ANAPH femme CONN 3DU pêcher
'Hier, il parlait à ces femmes qui pêchaient.' 


\section{Xe et la définitude de l'antécédent}

Le fait que la relation marquée par xe soit une identification ne signifie pas que le terme ainsi déterminé est toujours indéfini. Si un terme indéfini est toujours associé à une relative avec xe, comme en (11) où agu est indéfini, le terme constituant l'antécédent peut aussi être défini. En (12) thaamwa est déterminé par un pronom anaphorique : les femmes ont déjà été mentionnées, mais elles sont toutefois identifiés dans la relative comme celles qui pêchaient, d'où la présence de xe qui articule le segment thématique et le segment rhématique. Il faut donc dissocier la définitude de l'antécédent du type de relation construite entre l'antécédent et la relative : si la détermination est définitoire et identifie le terme, xe apparaît, s'il s'agit au contraire d'une information déjà connue, c'est un déterminant déictique ou anaphorique qui articule les segments et sert de connecteur. On peut ainsi opposer (14a)qui démarque un thème et un rhème et (14b) qui réfère à une détermination pré-construite, marquée par le pronom anaphorique bai. La traduction, par un attribut ou une épithète, tente de rendre compte de cette différence essentielle.

(14a) habruali-n xe hooli habuan xe hmwêên xa foro. vêtements-POss.3sG CONN ce.ANAPH vêtements CONNêtre grand aussie être blanc 'Ses vêtements, ce sont les vêtements qui sont grands et blancs.'

(14b) habuali-n xe hooli habuan bai hmwêên $x a$ foro. vêtements-Poss.38G CONN ce.ANAPH vêtements ce.ANAPHêtre grand aussie être blanc 'Ses vêtements, ce sont les vêtements grands et blancs.'

En (14a), xe a successivement deux de ses fonctions: thématisation de habwali-n et relateur qualifiant et identifiant le nom habwan; ou plutôt la même fonction récursive de démarcation entre un segment thématique et un segment rhématique constituant une identification. C'est en définitive le principe unique auquel xe est associé, qu'il s'agisse de thématisation, de relativisation, de complémentation et parfois même de subordination. On a donc une clé unique pour des opérations en apparence très diverses.

\section{Relatives avec déterminants déictiques ou anaphoriques}

\section{A) Exemples contrastifs}

a) Identification : $x e$.

Le thème est posé puis identifié (15a) :

(15a) na tu thanmwa an-xiik xe $i$ yeenare vhan nêlêmun. 1SG trouver femme CLASS-un CONN 3SG apprendre.TR parler nêlêmwa 'J'ai rencontré une femme qui apprend le nêlêmwa.' (ou) 'Jai rencontré une femme, elle apprend le nêlêmwa.'

b) Détermination préconstruite

Le déterminant post-nominal (déictique, directionnel ou anaphorique) est obligatoire, il s'accorde en nombre avec l'antécédent et fait fonction de connecteur pour construire la relative. Ainsi le déterminant bai est singulier en (15b) et le déterminant déictique mahleena est pluriel en(16). 
32 - Déterminant anaphorique : bai est l'anaphorique référant à du déjà connu, de l'expérience partagée.
(15b)
thaamua bai
(i) yeenare
vhaa nêlêmwa.
ISG trouver femme ce.ANAPH (3SG) apprendre.TR parler nêlêmwa 'J'ai rencontré la femme qui apprend le nêlêmwa.'

33 Contrairement à (15a) où le pronom sujet ne peut être effacé, en (15b), le pronom sujet est optionnel dans la relative s'il est coréférent avec l'antécédent, ce qui témoigne d'une relation étroite entre l'antécédent et la relative.

34 - Déterminant directionnel ou déictique : référence situationnelle.

\begin{tabular}{|l|l|l|l|l|l|l|l|}
\hline (16) & na & fawulu & hulak & mahleena $^{5}$ & (hla) & taabwa & mwena. \\
\hline & 1SG & parler & vieux homme & ces.DEICT & (3PL)s'asseoir & endroit.DEICT & \\
\hline \hline \multicolumn{6}{|l}{ 'J'ai parlé à ces vieux qui sont assis ici.' } \\
\hline
\end{tabular}

\section{B) Effacement ou maintien du pronom personnel sujet dans la relative}

35 - Le pronom sujet est optionnel dans la relative à détermination préconstruite s'il est coréférent avec l'antécédent [(16) \& (17)].

'Je connais l'homme qui a écrit cette lettre.' (litt. Je connais l'homme là, (il) a écrit cette lettre).

Dans certaines variétés d'anglais, les relatives sujet peuvent avoir une construction similaire sans relatif, on peut donc mettre en parallèle (17) na mwimwi agu bai (i) tii tiiwo hleny et «I know the man ø wrote this letter ».

37 - En revanche, si le pronom sujet n'est pas coréférent avec l'antécédent (19a), sa présence est obligatoire dans la relative. Son absence dans un tel énoncé construirait l'équivalent d'un passif avec un sens résultatif (19b), car dans cette langue sans passif, l'effacement du pronom sujet ou de l'agent nominal est l'une des stratégies équivalant à une 'passivation'. 
(18)

mwa-m

maison-POSS.2SG

\section{ho-iva?}

ce-où?

\section{'Quelle est ta maison ?'}

(19a)

$\begin{array}{llllll}\text { mwa } & \text { eli } & \text { hla } & \text { ya-ri } & o & \text { maaxi. } \\ \text { maison } & \text { ce.ANAPH } & \text { 3PL } & \text { couvrir-TR } & \text { PREP } & \text { paille }\end{array}$

'La maison qu'ils couvrent de paille.'
(19b) mwa eli
ya-ri
$o$
maaxi.
maison
ce.ANAPH
couvrir-TR
PREP
paille

'La maison couverte de paille.'

Le terme 'non actif' est toutefois préférable à 'passif' dans la mesure où le verbe garde un suffixe transitif(ya-ri) comme dans une construction active. Le sens non-actif résulte de l'effacement du pronom sujet hla et de l'absence d'agent.

Seules les relatives construites avec un déterminant déictique ou anaphorique permettent l'effacement, dans la relative, du pronom sujet coréférent avec l'antécédent ; les constructions avec xe ne le permettent pas, ce qui est cohérent avec leur fonction d'identification.

\section{Référence avérée vs hypothétique : xe/o dans les relatives thématiques}

Le morphème $x e$ a donc pour fonction essentielle de délimiter un thème et un rhème, i.e. le prédicat qui identifie ce thème. Outre sa fonction de démarcation, xe est aussi associé à l'identification et au mode assertif, il n'apparaît que dans des énoncés affirmatifs ou factifs référant à des faits avérés. Dans les énoncés interrogatifs, injonctifs, négatifs ou hypothétiques, xe commute avec la marque de l'hypothétique o (20a) \& (20b). C'est cette commutation qui tend à prouver qu'il est lié à une forme d'identification; s'il n'y a pas de référence possible, la marque du virtuel o le remplace.

41 - Enoncé assertif :
(20a) na
îyûlt
taraushi
xe
tadalan.
1SG acheter
pantalon
CONN être noir
'J'ai acheté un pantalon noir.' (litt. un pantalon qui est noir).

42 - Enoncé injonctif, hypothétique :

\begin{tabular}{|l|l|l|l|l|l|}
\hline$(20 b)$ & na-me & orop & o & ulo & shi-ny ${ }^{6}$. \\
\hline & donner-DIR & robe & VIRT & être rouge & côté-Poss.1SG \\
\hline & 'Donne-moi une robe rouge.' (litt. une robe qui soit rouge). \\
\hline
\end{tabular}

$43-$ Contexte négatif: 
Il n'y a pas de référence avérée, la relative est marquée par o (20c). Comparer avec (12).
(20c) kiya
agu
$o$
na
axi-e.
il n'y a pas
personne
VIRT 1SG
voir-3SG
'Je n'ai vu personne.' (litt. il n'y a personne que j'aie vu.)

(12) $f_{0}$
fo agu xe hla uya.
ily a gens CONN 3PL arriver
'Il y a des gens qui arrivent.' (ou) 'Il y a des gens, ils arrivent.'

$45-$ Contexte interrogatif, virtuel

$\begin{array}{llllll}\text { (20d) fo } & a g u k & o & i & \text { taau-ri } & \text { wany? } \\ \text { il y a personne } & \text { VIRT } & \text { 3SG attendre-TR bateau } \\ \text { 'Y a-t-il quelqu'un qui attend(e) le bateau ?' } & \end{array}$

\section{Variations xe / 0}

Il y a parfois des variations possibles entre xe et $o$ dans lesinjonctions, avec des sens différents; l'énoncé (21a) établit une reférence certaine; alors qu'en (21b) avec le morphème $o$, l'existence du couteau est hypothétique :
(21a) fle-dume
hele
$x e$
caak.
apporter-DIR
couteau
CONN
être aiguisé
'Apporte-moi un couteau aiguisé.' (existence certaine)
(21b) fhe-dume
hele
$o$
caak.
apporter-DIR couteau VIRT être aiguisé
'Apporte-moi un couteau aiguisé.' (existence hypothétique)

Le morphème o est aussi associé à la complémentation verbale et la subordination dans un contexte hypothétique, tout comme $x e$ dans les contextes assertifs référant à un fait avéré. Mais il y a toutefois une différence notable : à la différence de xe, le marqueur de l'hypothétique n'a jamais de fonction de thématisation, ce qui ne surprend pas si l'on considère que thématiser consiste à poser comme terme de départ un élément qui sert de repère et qui doit donc avoir une certaine stabilité référentielle.

Comparons l'énoncé virtuel (22), lié à la présence du futur et l'énoncé assertif (9) :

(22) io na xam gaa shayailî me uya da o na kûûtî FUT 1SG ASS CONT travailler.TR VISÉE arriver en haut VIRT 1SG finir.TR 'J'y travaillerai jusqu'à ce que j'aie fini.'
hla horêân
utya
da
xe taan.
3PL se reposer
arriver en haut
CONN être jour
'Ils se reposent jusqu'à l'aube.

Les cas suivants sont des exemples de complémentation verbale à sens hypothétique, marqués par o ; il s'agit de verbes à sens négatif (kuat 'refuser') ou associés à de l'incertain (khaxac 'hésiter'). 


\section{(23) $i$ kuat o $i$ yagei-e \\ 3SG refuser VIRT 3SG aider-3SG \\ 'Elle refuse qu'il l'aide.' (litt. elle refuse s'il l'aide).}

S'opposent ainsi la valeur hypothétique avec khaxac 'hésiter' en (24) et la valeur assertive avec kaxaak 'être certain' en (8) :

$\begin{array}{lllll}i & \text { khaxac } & o & i & \hat{a} \\ \text { 3SG hésiter } & \text { VIRT } & \text { 3SG } & \text { partir }\end{array}$

$\begin{array}{llllll}\text { na kaxaak } & x e & n a & \hat{a} & \text { Pum. } \\ \text { 1SG être sûr } & \text { CONN } & \text { 1SG } & \text { aller } & \text { Poum } \\ \text { 'Je suis sûre d'aller à Poum.' } & & & \end{array}$

51 La commutation de xe avec le marqueur de l'hypothétique dans certains contextes de détermination (relatives et complémentation) suggère donc qu'il a plus que des fonctions démarcatives et qu'il est aussi un marqueur d'hypotaxe.

\section{Traces résomptives}

52 Les morphèmes qui articulent les relatives ( $x e$, déterminant déictique ou anaphorique) ont un rôle essentiellement démarcatif, ils ne marquent pas la fonction syntaxique du terme déterminé dans la relative, comme le font les pronoms relatifs 'who(m)', 'whose' en anglais ou 'qui', 'que', 'dont' en français, qui cumulent diverses fonctions: celle de connecteur, d'anaphore et de marque de fonction syntaxique. Ici, ces fonctions sont décumulées sur des éléments différents. La fonction syntaxique est marquée par des traces résomptives in situ dans la relative ; ces traces permettent la coréférence du terme commun à la principale et à la relative. Ces traces sont les mêmes pour toutes les opérations impliquant le déplacement d'un terme de l'énoncé et sa reprise (i.e. thématisation, focalisation et relativisation). Du fait que le connecteur n'a qu'une fonction démarcative, il n'y a pas de restriction sur les fonctions susceptibles d'être relativisées. En voici quelques exemples.

53 - Object direct (patient).

(25) na mwimwi agu bai yo axi-e kôôbwan.

1SG connaître personne ce.ANAPH 2SG voir-3SG hier

'Je connais l'homme que tu as vu hier.' (litt. je connais l'homme tu as vu-lui hier)

54 - Complément circonstanciel de lieu.

55 La trace résomptive de la plupart des circonstants est le pronom locatif anaphorique le.

(26) $i$ ugn ni kuut pun-giik xe hla mu le ludak mulleedi.

396 aniver dans baie CLASS-une CONN 3PL rester LOC.ANAPH vieux cesANAPH

'Il arrive dans une crique où résident ces vieux.' (litt. il arrive dans une crique, ces vieux résident là.) 


\section{Construction des relatives et aspect}

On a montré les variations possibles de diathèse (active vs non active, résultative) liées à l'effacement du pronom sujet dans les relatives à détermination préconstruite (marquées par un déterminant déictique ou anaphorique), quand celui-ci n'est pas coréférent avec l'antécédent ( $\mathrm{cf} 3$.$) .$

Le recours à une nominalisation, apposée au terme déterminé, signale aussi une variation de diathèse et d'aspect, marquant l'aspect résultatif ou l'état résultant.

\begin{tabular}{|l|l|l|}
\hline Type de détermination & Antécedent & relative à détermination préconstruite \\
\hline Actif & Nom & + pronom sujet $+\mathrm{V}(\mathrm{cf}(19 \mathrm{a}))$ \\
\hline Résultatif & Nom & + pronom sujet effacé $+\mathrm{V}(\mathrm{cf}(19 \mathrm{~b}))$ \\
\hline Résultatif , non actif & Nom & + nominalisation $(s h \hat{a}-$, hna- $)+$ déterminant possessif \\
\hline
\end{tabular}

\section{Nominalisation et détermination résultative}

A la construction active (27a) avec un sujet qui est l'agent du verbe de la relative, s'oppose la construction nominalisée (27b) qui préserve la continuité thématique du patient en lui juxtaposant une détermination nominalisée, tandis que l'agent est exprimé sous forme de déterminant possessif. Divers préfixes nominalisants permettent ce type de construction: hna- 'lieu (de)', shâ-' 'contenu (de)'.

\section{Préfixe shâ- : détermination résultative}

shâ- est un préfixe collectif dérivé du nom shaget, il exprime une relation de contenant à contenu, tel que 'l'équipage' (d'un bateau), les 'habitants' (d'un pays), la 'prise' (à la pêche ou la chasse).

En (27b) la nominalisation a un sens résultatif ou exprime un état résultant, c'est l'équivalent d'une construction passive dans nos langues.
(27a) na khu-xi
mugic mahlaabai
i khîli
a nyanya.
1SG manger-TR bananes ces.ANAPH.PL 3SG cuire AGT maman
'Jai mangé les bananes que maman a cuites.'

61 En (27b), le terme déterminé (mugic) est le patient ; l'agent (nyanya) est syntaxiquement le déterminant possessif de la nominalisation; en l'absence de passif, la nominalisation exprime un état résultant.
(27b) na khu-xi
mugic
shâ-khîti-wo
i nyanya.
1SG manger-TR bananes COLL-cuire-COLL PREP maman
'Jai mangé les bananes cuites par maman.' (litt. les bananes résultat de la cuisson de maman). 


\begin{tabular}{|l|l|l|}
\hline Type d'énoncé & $\begin{array}{l}\text { relative à valeur } \\
\text { d'identification }\end{array}$ & $\begin{array}{l}\text { relative à détermination } \\
\text { préconstruite }\end{array}$ \\
\hline $\begin{array}{l}\text { Déclaratif ( référant à un fait } \\
\text { avéré) }\end{array}$ & $x e$ & $\begin{array}{l}\text { déterminant anaphorique ou } \\
\text { déictique }\end{array}$ \\
\hline $\begin{array}{l}\text { Non-asserté } \\
\text { négatif, virtuel) }\end{array}$ & $*$ \\
\hline \hline Déclaratif à sens résultatif & $*$ & shâ-+ patient / objet \\
\hline$*$ construction non attestée & &
\end{tabular}

\section{Eléments de comparaison} destribution » car elle apparaît dans des énoncés où l'on attribue une propriété au sujet (ex : « cet homme est le chef » ou « ce jeu est dur ») et qui sont donc de type définitoire et qualificatif ; ce morphème apparaît sous diverses formes selon les langues : $a, k a, x e$.

67 Mais seul le nêlêmwa $a^{7}$ semble avoir grammaticalisé ce morphème de thématisation en morphème plurifonctionnel (thématisation, relativisation, complémentation verbale de type factif),

68 3. ou enfin une détermination nominalisée avec une valeur aspectuelle résultative ou des fonctions de diathèse.

69 Rappelons enfin qu'il n'y a pas de distinction entre relatives restrictives et descriptives. L'équivalent d'une relative descriptive est exprimé soit par l'une des deux constructions de base ou encore par une construction paratactique (28) avec un pronom de rappel.
(28) $\quad$ in
perui ma
aaru thaamwa, hlinaidu haga moxmaidu.
1PL.EXCL rencontrer COMIT deux femme ces2 en bas pêcher en bas là 'Jai rencontré deux femmes, les deux qui pêchent là-bas.'

\section{Eléments de typologie}

Le lien entre la thématisation et la construction des relatives n'est pas rare dans les langues austronésiennes. 
71 - En chamorro (Micronésie, austronésien occidental), la thématisation, l'interrogation et la relativisation sont structurellement similaires : on pose le thème puis on prédique. (Wouk 1986:149)

72 - Dans les langues des Philippines, d'Indonésie, en malgache (Keenan 1976:265), on ne peut relativiser un terme que s'il est le «topic» de l'énoncé, i.e. un terme déterminé qui constitue le centre thématique de la phrase, marqué comme tel par un affixe de diathèse sur le verbe. Ainsi, la relativisation d'un « acteur / agent » requiert la construction dite « Actor topic ", celle d'un patient requiert la construction dite «Patient topic " (pour les faits en tagalog, voir Schachter 1976:500). En outre, un terme indéfini (non référentiel) ne peut être ni thématisé, ni relativisé (Schachter 1976:514).

73 - Sur ce même principe, en palau (Iles Palau, Micronésie, austronésien occidental), les relatives déterminant un «Acteur» / sujet ou un «Patient» / objet requièrent des constructions différentes (Hagège 1985, Lemaréchal 1991).

74 - Dans une relative sujet / Actor, la construction dite « indicative » apparaît, l'acteur est thématique (le connecteur est (e)l dans tous les cas) ; comparer (29a \& b) :

(29a) $A$ redil a s-il-seb-ii a alái.

ART woman burn-PERF-burn-3SG.OBJET ART house

'The woman has burned the house.' (Lemaréchal 1991:214).

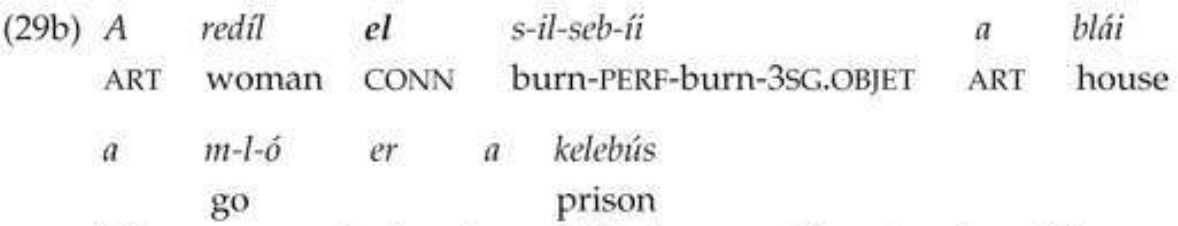

'The woman who has burned the house will go to prison.' (Lemaréchal 1991:115).

75 - Dans une relative objet / Patient, dont le Patient est thématique, apparaît alors la construction dite "passive-hypothétique ${ }^{8}$ " $(29 \mathrm{c}$ et $29 \mathrm{~d})$, qui est marquée par un paradigme de pronoms sujets spécifiques à cette construction et préfixés au verbe (le- en 29c. et d.).

L'énoncé (29c) se traduit par une construction «passive ", elle est en faitassociée à la thématisation du patient défini et à des pronoms préfixés au verbe (le-) référant à l'actant1 (et qui sont proches des marques possessives), mais le suffixe (-ii), qui réfère à l'objet, subsiste.
(29c) $A$
blái
a $\quad$ le-s-il-seb-ii
a redil.
ART house 3SG.PASS-burn-PERF-burn-3SG.OBJET ART woman

'The house was burned down by the woman.' (lit. the house, the woman burned it down) (Josephs 1975:453, cité dans Lemaréchal 1991:213).

La relative (29d) ne s'en distingue que par l'apparition du connecteurel :
(29d) $A$
blái
el le-s-il-seb-ii
a redil.
ART house CONN 3SG.PASS-burn-PERF-burn-3SG.OBJET ART woman 'The house that the woman burned down.' (Lemaréchal 1991:214).

Le patient est donc thématisé tandis que l'agent est coréférencé par des pronoms proches des formes possessives, ce qui évoque les constructions nominalisées du nêlêmwa en (27)à sens non actif et résultatif. 
79 - Il y a donc une corrélation, soulignée par Foley \&Van Valin (1985:317), entre la thématisation / topicalisation et la relativisation. Le terme constituant le centre thématique («focus ») de l'énoncé est coréférencé par un affixe de diathèse sur le verbe et joue le rôle de pivot syntaxique pour diverses opérations de détermination nominale (relatives) ou verbale (complémentation).

«The explanation for these Palauan data seems to be that a left-dislocation or topicalisation construction began to be used in relative clauses to meet the constraint that only topical NPs were relativizable. [...] It may also explain somewhat the situation in Tagalog and other Philippine languages. [...] the topic became the pivot for more and more of the syntactic processes [...]. » Foley \&Van Valin (1985:317)

80 - En outre, le connecteur des relatives - le connecteur multifonctionnel (e)l (Hagège 1986:116 ; Lemaréchal 1991:131) apparaît dans les constructions attributives et a aussi une fonction de subordination et de complémentation (Hagège 1986:115-133 ; Lemaréchal 1991:114-139).

Il y a là un parallèle intéressant avec les diverses fonctions du morphème xe en nêlêmwa, à la différence près que les relatives avec xe sont liées à l'identification du terme déterminé.

- Le système en nêlêmwa s'apparente davantage à ce que Justus (1976) décrit pour le hittite, où il n'y a pas de 'relatif' quand la détermination relative est préconstruite. En revanche, quand elle introduit une détermination nouvelle, la relative est marquée par un pronom $k u-/ ~ k w^{-9}$, pronom relatif-interrogatif-indéfini (Justus 1976:235).

Le morphème $k u$ - est démarcatif, à la charnière entre le thème et l'élément nouveau ; ce n'est qu'ultérieurement qu'il est analysé comme un relateur (Lehmann 1995:115) et comme marque de subordination (Justus 1976:225). Le caractère indéfini, non anaphorique du relatif $k u$-résulte de sa fonction de marqueur introduisant une information nouvelle (Justus 1976:238-39).

"Justus argues that the use of the relative-interrogative-indefinite pronoun $\mathrm{kw}$ - to mark the focal NP (i.e. introducing new information) in thematic clauses (i.e. containing old information) [...] is a characteristic of somewhat later Hittite texts. But even at this later stage, [...] $k u$ functions as the indefinite marker of a noun phrase in an independant clause rather than a "relative pronoun ». [...] what appears to be a correlative clause is in fact a topic clause that states a theme whose domain is not just the next clause but, potentially, several following clauses [...]. Eventually the $k w$-came to be understood as grammatically linking the theme clause to the following clause. [...] We have here an example of a relative clause construction which was originally not a grammatical entity but simply part of the way in which discourses are organised in a particular language coming to be grammaticalised as an embedded clause. " (Justus 1976, cité par Hopper et Traugott 1993:193-95).

84 - En Vieil-anglais, les relatives sont construites par un connecteur invariable ( $p e$ ) dont l'origine est déictique ${ }^{10}$; il délimite la principale et la relative sans indication casuelle et les relie dans une construction paratactique (30).

«Wiegand (1987) suggests that pewas originally a deictic marking marking the clause it introduces as a comment on or evaluation of the situation described in the preceding clause; [...].pewas later reinterpreted as a subordinator in various contexts (relative, complement, causal, etc.). » (Traugott 1992). 
(30) \& pa men comon on East Engle pe on pam anum scipe and the men came to East Anglia CONN on that one ship waron swide forwundode

were very wounded

traduction littérale : [and the men came to East Anglia] pe [on that sole ship were severely wounded]

'And the men who were on that sole (surviving) ship came to East Anglia severely wounded:

(ChronA [plummer] 897.51; O'Neil 1977:200, cité dans Hopper and Traugott 1993:192).

Noter l'absence de pronom personnel dans le deuxième segment après le relateur $p e$.

O'Neil (1977) montre que l'imbrication de la relative s'est faite par un mouvement à gauche, i.e. par thématisation / topicalisation.

"[...] relative clauses started out essentially as adjuncts, that is, as paratactic clauses close to the end of the sentence. (O'Neil) hypothesizes that their path to integration within the matrix clause was via a stage of topicalisation which moved certain relative clause structures to the left of the sentence; this is a stage of hypotaxis. Finally, clauses came to be embedded as dependent clauses immediately associated with their head nouns. " (O'Neil 1977, cité dans Hopper et Traugott 1993:190).

En (31), le sujet «our forbears » est thématisé, la relative est imbriquée et un pronom démonstratif anaphorique $(b a)$ - s'accordant en nombre avec l'antécédent - apparaît facultativement avant le connecteur (be).

Ure ieldran ba be pas stowa ar hioldon,
our forbears those CONN these places previouly held
hie lufodon wisdom
they loved $\quad$ wisdom

'Our forbears (those) who previously possessed these places, they loved wisdom.'

(c. 880, CP LetWerf 31; cited in O'Neil 1977:202, cited in Hopper and Traugott 1993:192).

Les fonctions de relateur et d'anaphore étaient donc dissociées en Vieil-anglais. Quant au connecteur déictique pe,il était aussi plurifonctionnel: «relatif», marqueur de complémentation et de subordination.

\section{Conclusion}

Il y a donc de très nombreux parallèles, qu'il s'agisse de l'origine des connecteurs (des articles, des démonstratifs ou des interrogatifs qui articulent un thème et un rhème en marquant un lien paratactique entre les segments) ou qu'il s'agisse de leur plurifonctionalité, ces connecteurs grammaticalisés en "relatifs» marquent ainsi diverses sortes de détermination, la complémentation verbale et la subordination.

Si l'on s'en tient à la morphologie des relatives, on ne peut que constater une extrême diversité ; on peut toutefois dégager des tendances et des types plus que des invariants. 
châssement implique l'hypotaxe et est associé à des connecteurs grammaticalisés, alors que le non enchâssement permet la parataxe et la présence de simples connecteurs avec un élément anaphorique. Ainsi, les relatives du Vieil-anglais avec leur connection lâche ont cédé la place à partir du Moyen-anglais au nouveau système des relatifs en wh-, issus des interrogatifs, eux-même issus des formes pronominales indéfinies en $k w$ - de l'Indo-Européen. Ces relateurs ( $q u$ - et wh-) sont des indicateurs généraux d'enchâssement et d'hypotaxe (subordination, complémentation) qui saturent la place d'un élément manquant et cumulent les fonctions de connecteur, d'anaphore et de marque de fonction syntaxique auparavant dissociées sur des marqueurs différents.

\section{BIBLIOGRAPHIE}

\section{Références (Nouvelle-Calédonie)}

BRIL, I. (1995) Analyse linguistique de la structure de l'énoncé dans la langue nêlêmwa, Thèse de Doctorat, Université Denis Diderot - Paris 7.

MOYSE-FAURIE, C. (1983) Le drehu, langue de Lifou (Iles Loyauté). Paris, SELAF.

- 1995, Le xârâcùù. Eléments de syntaxe. Peeters, LCP 10.

osumi, Midori (1995) Tinrin grammar. Oceanic Linguistics Special Publication, $N^{\circ} 25$, Honolulu : University of Hawai'i Press.

OZANNE-RIVIERRE, F. (1976) Le iaai, langue mélanésienne d'Ouvéa (Nouvelle-Calédonie). Paris, SELAF. RIVIERRE, J.C. (1980) La langue de Touho, phonologie et grammaire du cèmuhî (Nouvelle-Calédonie) Paris, SELAF.

\section{Bibliographie}

BOURCIER, G. (1977) Les propositions relatives en vieil-anglais, Paris, Ed. Honoré Champion.

CRÉPIN, A. (1978) Grammaire historique de l'anglais. Paris, PUF.

CUlioli, A. (1991) Pour une linguistique de l'énonciation.Opérations et représentations 1. Paris, Ophrys.

FISCHER, O. (1992) Syntax. In : The Cambridge History of the English language. Vol. II.

FOLEY, W.A., Van Valin, Robert D.Jr., Information packaging in the clause. In : Language typology and syntactic description, Vol. I (Clause Structure), T. Shopen (ed), 197-242. Cambridge University Press.

FUCHS, C., MILNER J. (1979) A propos des relatives. Paris, SELAF (NSP7).

FUCHS, C. \& al. (1987) Les types de relatives, Langages, décembre 1987, n·88, Larousse, Paris. 
HAGÈGE, C. (1986) La langue palau. Collection Forms of language structure, ed. by H. B. Rosén, Wilhelm Fink Verlag München.

HAIMAN, J. (1978) « Conditionals are topics », Language, 54, p. 564-589.

HOPPER, P.J. and TRAUGOTT, E.C. (1993) Grammaticalisation. Cambridge University Press.

JOSEPHS, L. S. (1975) Palauan reference grammar. The University Press of Hawai'i, Honolulu.

JUSTUS, C. (1976) « Relativisation and topicalisation in Hittite », in Li C.N. and Thompson S.A. ed, Subject and topic: a new typology of language, 213-246. New York, Academic Press.

KEENAN, E. L. (1976) « Remarkable subjects in Malagasy », in Li C.N. and Thompson S.A. ed, Subject and topic : a new typology of language, 213-246. New York, Academic Press.

KEENAN, E. L. and COMRIE, B. (1977) « Noun phrase accessibility and universal grammar », Linguistic Inquiry 8:63-99.

KEENAN, E. L. (1985) Relative clauses. InT. Shopen (ed.), Language Typology and Syntactic Description, Vol. II, 141-170. Cambridge University Press.

KUNO, S. (1976) « Subject, theme, and the speaker's empathy - A Reeaxamination of Relativisation Phenomena ", in Li C.N. and Thompson S.A. ed, Subject and topic : a new typology of language, 417-444, New York, Academic Press.

LEHMANN, C. (1995) Thoughts on grammaticalisation. LINCOM Studiesin Theoretical Linguistics 01. LINCOM EUROPA, München - Newcastle.

LEMARÉCHAL, A. (1991) Problèmes de sémantique et de syntaxe en palau, Editions du CNRS, Paris. MOSSÉ, F. (1946) Manuel de l'anglais du Moyen-Age, vieil-anglais. Aubier, Editions Montaigne, Paris. O'NEIL, W. (1977) «Clause adjunction in Old English », General Linguistics 17:199-211.

SCHACHTER, P. (1976) « The Subject in Philippine Languages : Topic, Actor, Actor-Topic, or none of the above ", in Li C.N. and Thompson S.A. ed, Subject and topic : a new typology of language, 491-518. New York, Academic Press.

TRAUGOTT, E.C. (1992) The Cambridge history of the English language, Vol I, Cambridge University Press.

\section{ANNEXES}

\section{Abréviations}

AGT marque d'agent ; ANAPH anaphorique ; ART article ; ASS marque assertive ; ASSOC associatif ; CLASS classifieur ; cOLL marque de collectif ; COMIT comitatif ; cONN connecteur ; CONT aspectcontinu ; DEICT déictique ; DIR directionnel ; EXCL exclusif ; FUT futur ; INCL inclusif ; INJ.NEG injonction négative ; LOC locatif ; NEG négation ; PERF perfectif ; PASS passif ; POSS possessif ; PREP préposition ; REL relateur ; TR transitif ; VIRT virtuel ; 3SG 3. personne singulier ; 3DU 3. personne duel ; 3PL 3. personne pluriel. 


\section{NOTES}

1. Les langues de Nouvelle-Calédonie appartiennent à la branche océanienne de la famille austronésienne. Le nêlêmwa, parlé dans l'extrême-nord de la Grande-Terre par un millier de locuteurs, est l'une des 28 langues de l'archipel.

2. Ainsi, en anglais, le relatif objet peut être omis à certaines conditions, de même que le relatif sujet dans certaines variétés d'anglais oral.

3. Le déictique hleny réfère à un élément proche du locuteur ; l'anaphorique baien (3) implique une référence partagée des co-énonciateurs et de ce fait est souvent associé au passé.

4. Voir Haiman 1978.

5. Le déictique -ena réfère à un élément à distance moyenne du locuteur.

6. Comparer avec la relative référant à une détermination préconstruite marquée par le déterminant déictique-ena :

$\begin{array}{lllll}\text { na } & \text { me } & \text { orow-ena } & \text { ulo } & \text { shi-ny } \\ \text { donner } & \text { DIR } & \text { robe-ce.DEICT } & \text { être rouge } & \text { côté-Poss.1sc }\end{array}$

'Donne moi la robe rouge.' (litt. ... la robe qui est rouge).

7. Deux autres langues, le tîrî (Osumi 1995 :240) et le iaai (Ozanne-Rivierre 1976) ont une construction relative analogue à une thématisation: en tîrî, 'the big tree that stands there ' s'interprète aussi comme 'the big tree, it stands there' (il n'y a pas de morphème de thématisation en tîrî, c'est l'intonation et l'inversion de l'ordre neutre qui indiquent la thématisation) ; en iaai, apparaît le morphème $a$.

8. Cette construction - qui marque l'orientation privilégiée du verbe vers le patient (sorte de GF) - apparaît dans divers types d'énoncés (Hagège $1986: 105,124-133$ et Lemaréchal 1991 :192-94) : 1) dans l'équivalent de subordonnées conditionnelles et temporelles ; 2) dans les énoncés injonctifs ; 3) avec des verbes dépendants de prédicats nominaux à valeur modale ('désir', 'vouloir, volonté', 'pouvoir', 'devoir') ; 4) dans les énoncés négatifs.

9. Dont sont issus les pronoms en qu-(langues latines) et ceux en wh- (langues germaniques).

10. pe et pæt étaient des déictiques; pæt fait partie du paradigme des pronoms se, seo,pæt; ce dernier donne « that » en anglais moderne.

\section{AUTEUR}

\section{ISABELLE BRIL}

Lacito-CNRS 